\title{
Ambient Assisted Living an Overview of Current Applications, End-Users and Acceptance
}

\author{
Robert Stodczyk, UHP Fischer-Hirchert* \\ Technisches Innovations management, Hochschule Harz, Wernigerode, Germany \\ *Corresponding author: Robert Stodczyk, Technisches Innovations Management, Hochschule Harz, Wernigerode, \\ Germany
}

ARTICLE INFO

Received: 幽 July 28, 2020

Published: 幽 September 15, 2020

Citation: Robert Stodczyk. Ambient Assisted Living an Overview of Current Applications, End-Usersand Acceptance. Biomed J Sci \& Tech Res 30(3)-2020. BJSTR. MS.ID.004949.

Keywords: Ambient Assisted Living; Assistive Technology; Active Assisted living; Applications; End-Users; Acceptancy; Overview; Review

\author{
ABSTRACT
}

Worldwide an ageing of the population can be observed. This imposes several problems on the concerning societies. There, a higher share of elderlies needs to be taken care of by a diminishing number in younger population. To still be able to provide sufficient care the use of technology is regarded as a solution. In this paper, we are examining the special field of Ambient Assisted Living (AAL) as an application of technology in health care. It mainly focusses on enabling independent living at home for people in need of care through technology. In detail, we aim at giving an overview of current applications, their end-users and their acceptance of AAL. We find that common applications of AAL are smart homes, sensors and robotics. The common end-user of these applications are elderlies. They have a positive opinion towards AAL in general but criticize their price and want to be included in the development process.

Abbreviations: AAL: Ambient Assisted Living; ICT: Communication Technologies; PIR: Passive infrared sensors ; BSN:body sensor networks; IADL: activities of daily living;EADL: enhanced activities of daily living; IADL: instrumental activities of daily living; IEC: International Electrotechnical Commission

\section{Introduction}

Ambient Assisted Living (AAL) is a research area, where the focus lies on enabling people with any kind of impairment to stay independent in their own home for as long as possible. To achieve this, Information and Communication Technologies (ICT) are used in various ways. The upcoming of Ambient Assisted Living can be regarded as the answer to several global trends. AAL technologies are expected to solve the problems that are imposed due to recent worldwide socio-economic developments. There, the ageing of the global population can be observed at first. Here, low birth rates stand opposite to a bigger share of elderly citizen with high life expectancies. At the same time, a decreasing supply in health care services results in rising costs for care. Also, a general increase of chronic diseases and the general will of elderly or impaired people to stay in their known environment puts pressure on the current health care systems of many nations. Therefore, Information and Communication Technologies have been a field of research and are expected to solve or to diminish the stated problems. Especially in the home environment, where AAL solutions are applied, the lack of formal caregivers can be decreased. Also, the visits to medics can be less frequently, because conditions might be detected early and thus, treatment costs can be decreased. The mentioned global developments increase the pressure on health care systems, the application of AAL technology is researched to ease these trends.

In this paper, we provide further background information on the causes that influenced the upcoming of Ambient Assisted Living in recent research as well as on the past evolution of these technologies. Furthermore, we conducted a literature review to give an overview about the following topics within the AAL domain. These topics are:

i. The current applications in the AAL domain are regarded with a focus on technologies that try to increase the independence of its users. We conclude that smart home technology, stationary and wearable sensors as well as different types of robots are of special interest in this domain. 
ii. The main end-users of these applications are investigated We observed that especially elderlies are addressed by AAL solutions in comparison to people with other kinds of impairments. Also, formal and informal caregivers are only targeted indirectly.

iii. The acceptance of Ambient Assisted Living products among its users is examined. We state that the attitude towards these devices is in general positive. Nevertheless, the price, data security, as well as an aversion towards certain sensor types is concerning. Also, user-centered design has become a main topic for increasing the devices' acceptance.

By showing the main applications for users of AAL,examining the main user groups and their acceptancy, this paper aims to provide a general overview of the Ambient Assisted Living domain.

\section{Background}

The upcoming of Ambient Assisted Living from a socioeconomic perspective as well its terminology and evolution are regarded in this section. Firstly, four socio-economic trends are described.

Angeing Population: According to the United Nations [1] the population is ageing worldwide. By 2050 it is prospected that the total number of people aged 65 and older will surpass the group of adolescents with an age between 15 and 24. This development is mainly a result of low fertility and mortality rates. Countries where these two factors apply are in the so called third phase of demographic transition. There the population growth only minimal or zero to negative. In comparison, the first phase is defined by low population growth due to high fertility and mortality rates. In the second phase has fast population growth, decreasing mortality and still high fertility. The third phase of this development can be seen in Europe, North America and Australia. It is even expected that Latin America and Asia will reach this phase until the end of 21st century [2]. An exception in Asia are Eastern and South-Eastern Asia, were the third phase is already expected in 2038. Here, Japan is specially to mention. From 2010 to 20192.6 million people died more than there were born. With less people born, a society is ageing[1]. At the same time the elderlies of today can show a higher life expectancy than former generations. In the EU women aged an average 84.3 years and males 77.7 years in 2015. Until 2060 the life expectancy for females is expected to rise an additional 6.2 years and for males 7.2 years [2]. An ageing population combined with higher life expectancy results in an increasing pressure on the rest of society.

Increasing Dependencies: In a society the non-working population is depended onthe working population. Therefore, the ageing of the society, higher life expectancies and lower fertility and consequently less workers are affecting a rise in old age dependency.The three factors which are stated above, cause that less workers must support an increasing share of elderly. This can be seen when comparing the so-called old-age dependency ratios. This ratio compares the number of elderly (aged 65 years or more) per one hundred people working (aged between 20 to 65 years). In the EU this number is constantly rising. In 1950 there were only 16 elderly per 100 workers, in the 2015 the number rose to 28 and for 2050 it is expected to reach 50[2]. This development is especially strong in Europe but some countries in Eastern- and South-EasternAsia are even expected to surpass a ratio of 50 [3]. As can be seen in (Figure 1) the ratio might be as high as 70.9 in Japan until 2050 according to IMF data.

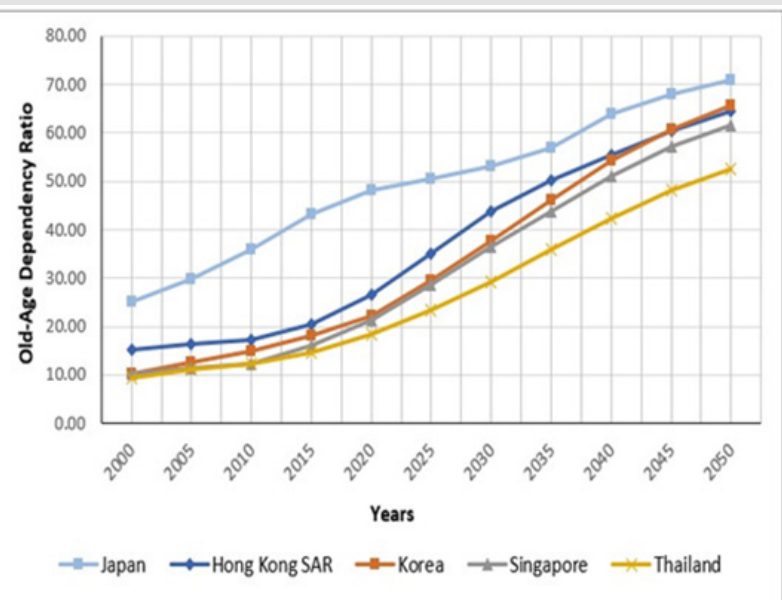

Figure 1: Old-Age Dependency in Asia according to [3].

A similar but less dramatic trend can be seen in the USA Canada and Australia. Here, the ratio will have surpassed 40 but stays below 50 until 2050 [4]. This trend of an increased need in support is further concerning, regarding how old age is typically spent. Even though the life expectancies are rising, this does not mean that these additional years are spent healthier. For example, in the EU women are living on average 64.2 years healthy followed by 19.4 years with disability. Males are living five years less on average and therefore 5 years less with disability. Also, an increase in chronic diseases like cancer and cardiovascular disease amongst elderlies or in mental illnesses can be observed. A growing number of elderlies with high life expectancies and a general rise in chronic diseases, challenges the supply of health care services [2].

Decreasing Supply in Health Care: The demand in health care services is rising as stated previously, but due to the decreasing working population, the supply might not be enough. The fall in working age population can above all be observed in countries in the third phase of demographic transition [5]. There, the increasing demand for health care enhances the burden on these service givers. Especially informal caregivers such as relatives are affected. In the EU they account for $80 \%$ of the supplied care. There, people aged between 50 to 74 years play an important role, since they are the primary caregivers to the oldest, with more than 85 years of age. But also, this group of people is shrinking [2]. Next to the decrease of informal care, also a general shortage in formal caregivers can 
be observed. This is not just due to the smaller share in workingage population but also the situation of the formal care sector. It is characterized with low working satisfaction, high fluctuation and few career opportunities. For example, in Germany these factors might add up to a shortage of 140.000 to 200.000 formal caregivers in 2025 [6]. A high demand in health care services that might not be supplied leads to rising costs for these services.

Higher Costs for Care: As a consequence, for higher demand and lower supply, costs are rising for health care. A longer living population with generally more diseases, requires health care services more often over their lifetime. At the same time in countries in the third phase of demographic transition, less people are paying into public health care systems. Where more people are indigent for care, but less people are financing it, the societies' overall financial burden increases [7]. Secondary to this, a general increase in health care costs can be observed. For example, the cost of cancer treatment rose from 3036 pounds in 1995-1999 to 35383 pounds in 2010-2014 [2]. The described demographic situation accompanied with a general rise in treatment costs influence an overall increase in health care costs.

Ageing at Home: Due to the described demographic trends more health care is demanded and needed, especially for the elderly. Nevertheless, people in need of care are preferring ambulant over stationary care. Being in a stationary care institution is often connected with a loss in independency by patients. Whereas in a familiar home environment people feel more responsible for themselves. The feeling of still being able to make own decisions to some extent is much valued by most of the elderly. This trend of "ageing in place" is also preferable for service providers. Being in a care residency is more costly not only for the patient. Also, the care at home can be more appropriate and easier provided and coordinated by the service [2]

Terminology: The term Ambient Assisted Living was first mentioned within framework programs for research of the European Union in 2004, still no universal definition of this topic was adopted yet [8]. Therefore, a conception of the AAL domain can be achieved by distinguishing it from other ICT and health related research as well as by looking at its evolution. The concept of Ambient Assisted Living can be regarded as a special field of application in the digitization of health care, the so-called e-health. ICT in the field of e-health can be applied in different forms. For example, health telematic uses telecommunication and informatics to communicate with remote patients, whereas telemedicine uses these technologies to give concrete health services [9]. In contrast, Ambient Assisted Living focusses on the users' domestic environment. While using assistive technologies the resident should be enabled to live independently in his home. There, the AAL solutions can range from the housing itself, the infrastructure such as sensors and actuators to health care services. It is due to this diverse possibility of how the resident can be supported by ICT, that no universal definition of AAL was adopted yet [10]. A common view looks at the emergence of Ambient Assisted Living technologies as the result of the evolution of general assistive technologies due to technological progress. Assistive technologies can be any device or system that enables the user to live independently in his home by supporting tasks the person would not be able to do on his own. These devices or systems can be simple sticks as walking aids, wheelchairs, adjustable beds or alarms for home security [11]. With the general technological progress these technologies became more advanced.

Especially, the development of ambient intelligence in combination with assistive technologies played a vital role in the upcoming of AAL. It uses recent advances in information technology to create digital environments that act as an electronic butler to the resident. To achieve this, ambient intelligence integrates sensor networks, to collect user and environmental data. The collected data is reasoned by the system to then perform actions which benefit the resident proactively in their daily life but in an unobtrusive manner [12]. If this paradigm of context aware computing, that integrates undistinguishable into the environment is used on the development of assistive technologies, this is called Ambient Assisted Living [13]. This view on the nature of AAL devices is especially adopted today because it makes use of the recent technological advances for example in artificial intelligence, machine learning or ubiquitous computing.

Ambient intelligence is the most recent paradigm of Ambient Assisted Living technologies. But before that AAL devices also existed. The emergence of Ambient Assisted Living technologies can generally be categorized into three generations [14].

i. The first AAL systems focused mainly on alarms in the form of wearable buttons. In the case of an emergency this button can be pressed by the user to call for help.

ii. In the second generation of devices the systems should not be dependent on the users' interaction but rather detect an emergency on their own. By using sensors, e.g. falls or gas leaks can be detected automatically.

iii. The third generation focusses not only on the detection and report of an incident but tries to prevent them.

Here the paradigm of ambient intelligence comes into use.

Ambient Assisted Living mainly differs from other e- health technologies in its focus on increasing the independence of its users' in their home. AAL technologies developed over three generations, where the most recent benefits from the upcoming of ambient intelligence. Research in Ambient Assisted Living has also become of global interest because of the stated socio-economic factors. After this background on AAL is given the following sections of this paper aim to give an overview of common applications of this technology, its user as well as their acceptancy of the devices. 


\section{Materials and Methods}

In this paper we aim at giving an overview of relevant topics within the AAL domain. Therefore, three relevant topics have been defined and formulated as the research questions of this paper. These are:

RQ1 What are the current applications of Ambient Assisted Living?

RQ2 Which end-users are the target group of Ambient Assisted Living applications?

RQ3 How is the acceptance of Ambient Assisted Living among its end-users?

To provide this overview we conducted a literature review in the following search engines:IEEE, PubMed, GoogleScholar and ScienceDirect. For RQ1 only literature, that fulfilled the criteria: published between 2015 and 2020; includes at least one of the terms Ambient Assisted Living, Active Assisted Living, Assistive Technologies, review, overview has been selected as primary literature. Supporting, secondary literature has been selected based on the primary or through free searches in the stated engines. To answer RQ2 and RQ3 the primary literature was used or also a free search was conducted.In this paper, we aim to give an overview of AAL by reviewing overviews of this domain. Thus, it is possible to identify relevant topics that occur in these different publications. In this way, this paper differs from other overviews that review individual AAL projects to identify trends or from publications providing a list of AAL projects that is limited to several topics.

\section{Results and Discussion}

In this section, we provide the results of the literature review for the individual research questions.

\section{RQ1 What are the current applications of Ambient Assisted Living?}

For the answer of the first research question several overviews of the AAL domain have been reviewed. Even though the applications of AAL to enable independent living for its user are diverse, three main topics were identified. These are Smart Home, Sensing and Robotics. Within these topics several approaches exist. These are highlighted through project examples in this paper.

Smart Home: In the regarded overviews the smart home technology is often described as an AAL application since it enables independent living in the domestic environment.As a reason for this [15] states the diminishing health status of elderly or disabled people overtime, that is accompanied with the wish to stay independent in a familiar surrounding. Therefore, ICT solutions that integrate into the home provide a solution. [16] has even observed that within the AAL domain special home accessories and furniture are the most common devices. For that reason, also the smart home technology can be regarded as a major contributor to Ambient Assisted Living to enable more independency for its users. The importance of this technology can be also seen as the digitization of the domestic environment becomes more and more a point of focus for health management of patients [12]. In [17] the smart home is described as living environment that is digitized by sensors and smart appliances and thus forms a network that is capable to deliver automated services to the user based on his lifestyle. In order to deliver that services, the smart home uses various devices to firstly monitor the activities of the resident. The smart home is then able to analyze the collected data about the resident's activities in his environment. Based on this analysis the digital environment can offer services tailored to the resident and assist him in his daily life. This is achieved through various components: sensors (e. g. motion sensors), household appliances (e.g. lights), actuators (e.g. door openers), security (e.g. password locks), communication (e.g.human machine interfaces).

The independence of a smart home resident in AAL can be achieved in various ways. A typical application in this context is described in [15] and can be seen in (Figure 2). Here the elderly home is equipped with a sensor network that monitors the resident. The sensor data is transmitted via WiFi to a base station that connects via a Gateway to the homes of peers, family or friends and to health care responsibles such as carers and doctors. In this way the health status of the resident can be continuously supervised. In case of an incident or emergency fast care can be provided, via direct communication among the participants. Al Shaqi $\mathrm{R}$ et al. describes three general categories that distinguish the ways how the resident of a smart home is assisted in his daily life e.g. through actuators or bycontributing to health care management. These three categories of AAL smart homes are:

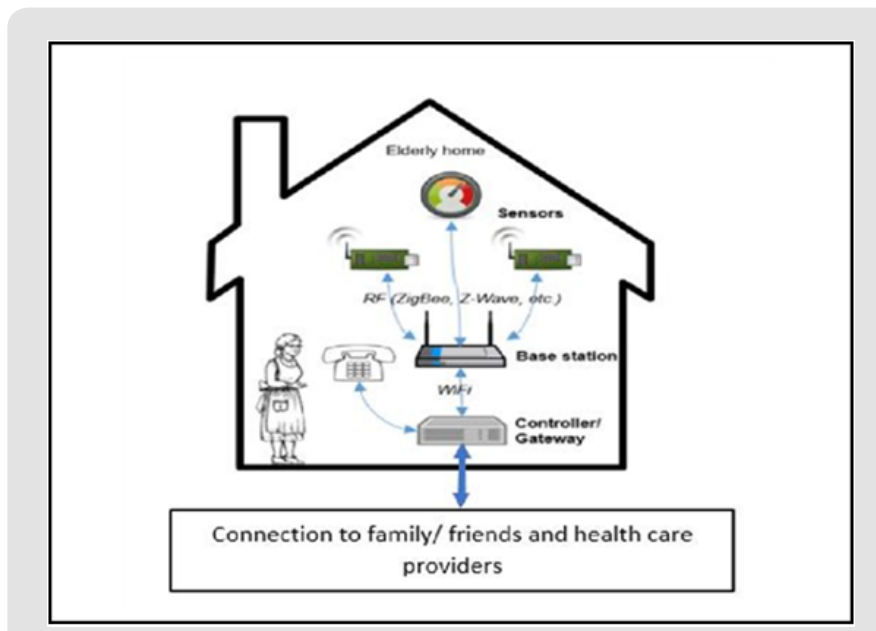

Figure 2: Typical Smart Home according to [15].

i. Smart homes that are assisting daily and social activities for example through providing orientation aid such as in [18].

ii. Smart homes that are improving the resident's safety for instance by predicting falls as in [19]. 
iii. Smart homes that asses the resident's health status through his vital parameters such as in [20]. The smart homes' ability to control daily tasks is further described in [14].

The two components are distinguished. First, there is the general health monitoring which comprises the systems and devices used to assess the inhabitants' health status. As a second, there are platforms that aim to integrate the several health systems, ensure interoperability and thus create the smart home environment. According to the premise of AAL the assistance of theresident through smart homes must be intelligent, unobtrusive and ubiquitous [10]. This non-disrupting nature of domestic monitoring systems is also emphasized in [21,22]. The medical status can be accessed by the responsible at any time without diminishing the user's independence and interrupting him in his daily living. At the same time the users stay connected and can communicate with the monitoring entity in case of need and vice versa.

To provide the communication between the various entities (sensors, actuators, people) in a smart home the Internet of Things technology is the enabler for home assistance. As described in Pawar $\mathrm{AB}$ et al., this technology interconnects sensing objects in the environment such as sensors to computational elements that evaluate the collected data. A service element then offers the corresponding assistance to resident. Concerning AAL, the Internet of Things technology can monitor single and multiple conditions of the resident such as changing heart rates or assist with medication management. Therefore, IoT for AAL can be seen as a problem solver in the medical home environment. Worldwide there are numerous projects regardingthe smart home technology in the field of Ambient Assisted Living. In the following three projects from Europe, America and Asia are presented.

A. CoachMyLife (Europe):[18]

i. Directed at elderlies to assist with daily activities at home

ii. Senior is equipped with smart watch, Bluetooth beacons are deployed in the home

iii. Machine learning is used to identify the desired task based on the senior's position to the beacons

iv. Help to fulfill the task is sent to a tablet in form of single steps

B. Vital Radio (America):[20]

i. Smart home environment, monitors the inhabitant's breathing and heart rate remotely without body contact

ii. By analyzing variations of wireless signals through persons chest and skin vibrations

iii. Assessing health status unobtrusively

C. TRON Intelligent House (Asia):[24] i. Japanese effort to develop the computer-based society of the $21^{\text {st }}$ century.

ii. House equipped with 380 computers

iii. Used the TRON architecture to interconnect the computer devices.

iv. Several AAL applications: e.g. a toilet that can analyze the urine and blood pressure, motion sensors for lightning to prevent falls.

The smart home technology monitors the resident and provides custom services to him. In the AAL environment especially the assistance with daily activities, safety support, health monitoring and the unobtrusive nature of smart homes are relevant.

Sensing: Sensing is a vital part in Ambient Assisted Living to gather information about the elderly or incapacitated. This information is useful when examining the health status through vital signs, monitoring the inhabitant's daily life or preventing accidents such as falls. A diversity of available sensors forms the basis of an Ambient Assisted Living environment. For that reason, the different sensors and their combination is a topic discussed in AAL reviews.The sensing element in Ambient Assisted Living is of special importance because it enables the monitoring of a person without the need for his intervention. This represents an advantage of AAL traditional health care devices that are often too difficult to use for impaired people. a major advantage [17]. There, the monitoring task can be accomplished by a wide variety of sensors.In [25] two general categories of sensors are distinguished. These are static sensors to monitor the user from a fixed location and mobile sensors that can also be worn. Their overall aim is to recognize human activity which is then processed to provide AAL services.

According to this study, the various sensors are commonly used for home safety, home automation, activity monitoring, fall detection, localization and tracking and health status monitoring. This study also provides an overview of sensors that are widely used in the Ambient Assisted Living domain.

Therefore, three different types of sensors are distinguished. These are:

a. Passive infrared sensors (PIR). These are usually fixed at a certain position. They are able to detect human motion in the environment. Thereby it is possible for example to analyze the human activity levels, detect deviations from normal behavior or intruders. The PIR sensor can also be used to recognize if a person has fallen.

b. Vibration and acoustic sensors. There, Vibration is monitored by accelerometers, which are usually used as wearable fall detection devices. Nevertheless, vibration sensors can also be static e.g. by implementing them into the flooring. In 
that way they are to analyze floor vibration and detect activities such as walking or running. If a fall occurs this is also detected. Also, acoustic sensors can be used for that reason.

c. Camera sensors. These systems are like the others able to detect certain activities of the users' and abnormalities to them. In contrast to the other sensors, cameras are able to supervise a wide range of activities. Cameras are commonly deployed in a fixed position, which usually raises privacy concerns.

Instead the use of wearable body cameras is also possible. In this way, pictures of the user are not directly made whereas the detection of e.g. falls is practicable. [15] also distinguishes these three different ways of sensing either through wearable devices such as accelerometers, through mostly static motion systems or through vision systems that are able to classify activities based on video segments. Furthermore, it describes the sensing element as a sub-system of a activity recognition system. Furthermore, it distinguishes between direct sensing, which concerns the monitored person and between indirect sensing which focusses on the environmental parameters. Also different measurement types to the user are distinguishable.

According to Cedillo, P. et al. the most common devices focus on measuring the heartbeat, blood pressure, temperature or falls. A list with various sensor types and their measurement that are either ambient and in a smart home or wearable and mobile is also given in [13]. Especially wearable sensors are of interest in current

AAL applications. Because of their miniaturization they can be integrated seamlessly for example into the clothing of a person and thus enable unobtrusive monitoring. In contrast to other mobile sensors such as in smart phones the sensing here becomes invisible. By integrating a variety of such miniature sensors on the user's body so called body sensor networks (BSN) can be implemented. Thus, it is possible to asses a holistic health picture of an AAL user[22]. Next to health monitoring, [14] describes fall detection systems and the unobtrusive monitoring of daily activities as important applications of wearable sensors within the AAL domain. The applications of sensors in AAL are also diverse.They can be part of a smart home or be worn directly on the body. In following three projects are presented that use different sensor types to monitor the AAL user.

A. ALMA (Europe):[26]

i. targets people with mobility impairments and offers navigation and orientation help.

ii. uses radio frequency emitters for localization of people and objects, also smart cameras for indoor and outdoor localization, environmental monitoring and for assessing situations.

B. Bioharness (America):[27]

i. lightweight wearable system, carried around the chest ii. monitors several physiological parameters such as posture, acceleration or ECG.

iii. offers possibility to view the data live or log it

iv. used in an AAL environment to monitor vital parameters.

C. Ubiquitous home (Asia):[28]

i. Japanese smart home project

ii. monitors the resident by using a variety of sensors

iii. passive infrared (PIR) sensors, cameras, microphones, pressure sensors, and radiofrequency identification (RFID).

Sensing is vital for AAL. It monitors the AAL user and provides services based on this data. Sensing is achieved through wearable or static sensors. These can either monitor the person directly or indirect through the environment. Particularly the miniaturization of sensors enables unobtrusive monitoring. Common measurements of these sensors are heartbeat, blood pressure, temperature or falls.

Robotics: In recent years also the deployment of robotics in the Ambient Assisted Living environment was thematized. These robots can assist in various ways. The applications can range from the assistance with the tasks of daily living to improving the social interactions or provide entertainment. There, [17] gives an overview of the diverse possibilities how robots can be integrated to aid in the life of impaired people. In general, the robots are able to address physical, social or cognitive impairments. In these fields the most common solutions focus on mobility and selfmaintenance for example on feeding, bathing and grooming. These service robots help incapacitated with functional daily activities and thus ensure their independence. Other service robots provide cognitive aid for example by reminding the user to take the correct medicine or to follow a diet. Additional to safety and purposes, some robots are also designed for security and emergency intervention.

They are able to assess risk situations for example when a fall has occurred and then notify a caregiver. Companion robots are researched to improve a patient's wellbeing through humanmachine-interaction. In this way, for example people with a kind of cognitive impairment can be stimulated through the interaction with the robot. The study names another group of robots that provide social, educational or entertainment services. These group is called interactive simulation robots and focusses on immersive and realistic user experiences. [10] also takes up the importance of companion robots on people with cognitive impairments. They might not cure these impairments but offer stimulation through personal health management, information provision and entertainment. In [22] robots are mentioned as one of the most significant developments inassistive technologies. Particularly, their ability to monitor and engage people in social activities is stated. Also, [15] describes several projects in Ambient Assisted Living that are using robots. The document mentions 
for example robots to move objects or presenting food which in general assist functional daily activities. Others can enhance the communication between the person and a caregiver through the robot or improve social activities and entertainment. [14] has reviewed robotic projects in the AAL domain and classified them into several categories based on their assistive focus.

The first category comprises service and companion robots that serve as Electronical Aids to Daily Living (EADLs). These robots can assist with specific tasks such as bathing or serve as reminders for activities such as following a diet. This category also includes cognitive orthotic systems that can help with social interactions. Further categories include health robots e.g. for managing health data and intelligent physical movement aids.

In contrast to this [13] advocates a different categorization of robots based on the complexity of the task they are assisting with.

i. On the first level robots help with general activities of daily living (ADL). Most are designed to move objects or for feeding, bathing, grooming tasks.

ii. More complex robot systems care about instrumental activities of daily living (IADL) such as housekeeping, shopping or meal preparation.

iii. The third level comprises service and companion robots giving aid with enhanced activities of daily living (EADL) like hobbies and learning. Here service robots aim to improve interaction and companion robots the emotional wellbeing by offering companionship.

Also, through robotics the AAL user can be assisted indiverse ways. Different types of impairments can be addressed and tasks with different levels of complexity can be supported. In the following also three AAL robotic projects are presented.

\section{A. ALIAS (Europe): [29]}

i. robotic system for elderly living alone

ii. monitor users, provide cognitive assistance, and promote social inclusion

iii. observes acceptance of the robot by the user, given a cognitive user interface

iv. robot's behavior is proactive ensuring the user stays engaged in his surroundings

v. training and preservation of the user's mental functions

B. Pearl Robot (America): [30]

i. robotic system providing its users' cognitive assistance in form of reminders and mobility aid

ii. uses speech synthesis, a display and a moving head for user interaction
C. Paro (Asia): [31]
i. Japanese therapeutic robot in form of a white seal
ii. aims to bring the benefits of animal therapy to a domestic environment

iii. Paro's lively behavior reduces the stress of patients and caregivers, stimulates interaction between them.

iv. improves the user overall psychological wellbeing Robots in the AAL domain are able to assist users with physical, social and cognitive impairments. Various types of robots are existing that might function as aids to special activities, as cognitive aids or as health managers. Based on the complexity of the assisting task robots for general activities of daily living, instrumental activities of daily living and enhanced activities of daily living are distinguished.

\section{RQ2 Which end-users are the target group of Ambient Assisted Living applications?}

Ambient Assisted Living solutions in general are able to restore the independence of people anywhere it is lost. Still the current research and applications are evolving mostly around the specific target group of elderly. In the following section we provide insight into targeted user groups of AAL devices and which loss of independence makes a deployment of AAL devices useful.The socio-economic developments of an ageing society, which imposes a higher burden on younger generations, the increasing costs for health care accompanied with a general increase in age-related diseases, demands for solutions. These problems that especially developed regions such as North America and Europe are facing are to be dealt with the use of information and communication technologies. Therefore, Ambient Assisted Living technologies receive tremendous funding in the view of providing solutions for an ageing society. Even though these systems are not necessarily exclusive to elderlies with any kind of impairment they are mostly designed for them.

As it is stated in [16] the majority of AAL projects focusses on the elderly as the end user, only a minority concerns disabled, children, pregnant women or others. Also, [8] which gives an overview of the current situation in AAL research, describes that the demographic change is a key driver of AAL developments.In past projects particularly the safety of seniors has been addressed for example through fall detection systems. Even though there is a diversity of Ambient Assisted Living solutions only a few have found a wide adoption. The reason for this is, that the target group of AAL systems, the elderlies does not see a purpose to integrate these products in their life. Therefore, it states that already younger people must be a target group of AAL developments. Generally, Ambient Assisted Living products can also be used by younger and not impaired people to assist their lifestyle for example for fitness tracking or typical smart home applications. The early adoption 
of these solutions makes it easier to use them later in a seniorcentered context. There, for instance fitness trackers can serve as health monitoring devices for caregivers or automatic light controls can provide orientation at night.

According to Cedillo, P. et al. the end-user, mostly elderlies, are the main focus of AAL developments. Still, caring relatives or professional caregivers are a potential target group of Ambient Assisted Living products. Especially informal caregivers such as relatives provide a big pillar in health care systems. Therefore, technologies that provide assistance to incapacitated are indirectly benefitting informal caregivers. The reduction of their burden through assistive technologies enables impaired people to stay longer independent in their home. This holds also true for care professionals. Regarding a general shortage of this personal, AAL technologies are able to provide relief [32]. Next to looking at the target group of current AALsolutions, it is important to regard the degree of independence loss, that would make the use of these systems rational. Usually, the independence of a person is assessed through an observation of the activities of daily living Whether a person can live on his own is thus dependent on the level of assistance he needs with daily tasks like bathing or eating [33]. Lists of activities that are essential for daily life have been proposed by several contributions. They do not only regard the field of Ambient Assisted Living but also the need for care in general [34-36]. Commonly these activities consist of two groups. The independence assessment based on activities has been extended by Lawton and Brody in 1969 for the instrumental activities of daily living (IADL).

Similarly, to the Barthel Index it contains a scoring list to assess a person's independence. This time it includes functional skills necessary to live in a community. The listed activities are more complex than the basic activities of daily living and account for tasks such as using a telephone, shopping, housekeeping and the ability to handle finances [37]. Based on the assessment of these two types of activities the general need for care of a person is determined. In many health care systems these assessments decide over a person's extent of access to health care services and support. This might also decide over the access to Ambient Assisted Living technologies as most people are not able to afford these systems on their own and rely on the national health care system. [32] argues that to enable an independent life at home, the observation of usual and regular activities is not sufficient. Instead, he proposes to assess the cognitive and communicative functions of a person or for example the ability to decide on a daily routine by himself.

This proposal widens the term of care neediness. In this way Ambient Assisted Living products could be accessible for a broader audience. So, could for example companion robots be accessible for people with only mild cognitive impairments. The International Electrotechnical Commission (IEC) has developed several use cases for assisted living technologies and has mapped them to a person's level of need.

i. On the first level also independent living persons with no need for care are proposed as a potential target group of AAL. For example, by assisting the self-management of the health status.

ii. On the level where some living assistance is needed the AAL system is supervised by the user. It only occasionally interferes for example in case of a severe emergency.

iii. The next level concerns the instrumental activities of daily living. There, permanent assistance is needed but also the users' interaction.

iv. The highest level of assistance regards the basic activities of daily living. In this case the system should be able to act autonomous. The monitoring of activities then is not enough, active assistance is needed.

This proposal also mainly concerns usual and repeating activities and not directly mentions cognitive or psychological impairments. Still, it states that a deployment of AAL technologies would also be beneficial for people with no impairment for selfmanagement purposes or in emergency situations. Thus, this view goes beyond the general assessment of need for care [38].

In conclusion, the main target group of AAL systems is the elderly end user. In fewer cases the end users were disabled, children or pregnant women. Formal and informal caregivers are not the primary focus of Ambient Assisted Technologies, still they benefit from them indirectly. Nevertheless, AAL devices are not exclusive to a certain user group but depend on the assessment of need for care. This assessment is usually based on the need for help with basic activities of daily living or instrumental activities of daily living. But also, people with the need for communicative or social aid might be users of AAL technologies. Furthermore, the deployment of AAL products for people without any kind of impairment is thinkable for example to increase the acceptance of these technologies once an impairment has occurred.

\section{RQ3 How is the acceptance of Ambient Assisted Living among its end-users?}

The wide adoption of AAL systems is highly depended on the acceptancy of these systems by the end-user. This is especially of importance since the target group of AAL consists mostly of seniors. With advancing age, the use ofnew technologies imposes increasing difficulties on elderlies. Therefore, the development of AAL devices must be centered on the end-user's perspective. Already in 1999, Cowan D and Turner Smith A stated that assistive technologies in general are not adopted by the user because they impose a great burden. This burden can also be socially, because the devices are seen as not desirable in the user's social environment. He therefore states that the development of assistive technologies should be based on the want of the user and not just the need from a medical 
perspective. Next to this, the acceptance of AAL products as a part of assistive technologies depends on various aspects. Weiß, C. et al. describes several of them that apply for general innovations and assistive technologies. It states that the overall acceptance of a product is on the one hand determined by the general attitude towards a product for example regarding its cost.

On the other hand, it is determined by the customers' specific behavior with the product. Concerning the acceptance of assistive technologies, it refers to McCreadie and Tinker. According to them, the users' feeling toward these products is influenced by:

i. their subjective need for care

ii. their supply and costs

iii. the product properties.

Furthermore, a positive user experience, a compelling design and the integration into the personal space decides upon a products acceptance. Another important aspect is the volume of learning that is required for the products handling. Ambient Assisted Living devices achieved an overall positive feedback by surveying its target group. This is due to the hope for independence and safety that many people are associating with this kind of technology. But, a major aspect for especially older people is the cost-benefit ratio of AAL products. There, in general the willingness towards these products increases, if the subjective need for assistance rises. Also, a high accessibility and a low price as well as a reliable functioning of the devices are beneficial.Even though the elderly is the main focus group of Ambient Assisted Living solutions, particularly in a care related environment the acceptance of informal and formal caregiver is of importance. There, for professional caregivers the acceptance is lower in general. Many of them regard the technologies as anti-human in the care context, where human interaction is valued much. Also, they are concerned with the fear of losing their jobs to automation [32].

The highest acceptancy towards AAL was gained by safety and wellness products such as intruder detections. Low acceptancy was given to products which use vision-based sensors or microphones within the home. Although the general willingness to deploy AAL devices is high by elderly, they fear decreasing interactions with other people as a result of these technologies. Also, the data security of the users' is a topic of concern. The past developments in AAL have been exclusively done by engineers. Therefore, single products were not appealing for the seniors in contrast to the positive feeling towards AAL in general. As a result, recent developments are particularly developed together with the elderly end-user. Through this way of user-centric design, AAL developments are becoming more attractive for the target group. A broader adoption of AAL solutions is therefore possible. In [8] an acceptancy study for AAL devices is presented. It was conducted in Germany, where people with an age between 48 and 84 years have been questioned. The survey described several typical features of AAL systems concerning for example general functions or the ways of data transmission.

As a conclusion the study also presents an overall positive attitude towards Ambient Assisted Living technologies among the interviewed. Especially positive was the feedback for safety functions such as intruder detections. There, the questioned were willing to pay more than for wellness functions. Nevertheless, the overall willingness to pay for AAL devices is low. Also, low maintenance costs and energy consumption are demanded. Furthermore, the study concludes that the questioned do not want to align their daily routine according to these systems. They are also skeptical if a device can identify its user or the threat of data fraud. Comparing the different technologies, the interviewed were less skeptical towards mobile devices followed by stationary ones. Whereas, systems that are camera-based were refused. People that have already used AAL devices also stated, that they were confused with their operation to some extent.

Further constraints that are hindering a broader deployment of AAL technologies are listed in [22]. They comprise: the lack of skills to use the devices, the uncertainty about its benefits, disabilities that might hinder the intended use and the high costs of some systems. Also, data security is a concern of AAL users. Even though these constraints exist today, [39] states that coming generations will have other technical capabilities and thus, their acceptance of AAL devices might be higher than today. For that reason, still it is necessary to examine how AAL devices need to be designed around the user and which properties are perceived most enjoyable to him. In [40] the acceptancy of different ambient notification systems has been regarded to asses which systems are least obtrusive and favorable in their users' daily life. There, the projects aim is in general to support the AAL users' daily activities by giving assistance through notifications, e.g. a reminder to drink something. There, warmth as a form of notification was used in a scarf and rated especially high, because it provided a positive feeling. The use of light as a notification to water plants was also rated high, because the light was used in a non-obtrusive way. Auditive signals received less acceptancy because they reminded of alarms. Using ambient sounds such as bird noises as notification was rated higher instead.

[41] presents a study of acceptancy for a user interface that is used in the AAL environment to control several functions of a smart home. The system is a touchscreen PC with an internet connection. The user interface has been developed together with the future users, which are mainly elderlies. Also, the system was given the human-like name PAUL. Because of the easy to use interface and the humanization of the system it is regarded more as roommate than a technical system. For that reason, also the acceptancy of the connected AAL services is not regarded as obtrusive or watching. The acceptance of AAL devices is dependent on various factors. Especially, their subjective perceived use and the price are decisive 
for the adoption of an AAL device. Older people are in general positive towards AAL, because of a hope for more independence. Still the willingness to pay is not given. Even though AAL might also ease the life of care givers, informal caregivers are fearing diminishing humaninteraction due to this technology.

Among the mostly elderly end-users particularly security devices were rated positive. As a constraint to the broader adoption of AAL the focus on the mere need for care during the products' development is to mention. Instead a focus on the end-users' perspective should be adopted. The example of PAUL [41] shows that usercentered design makes it possible that the technology can be perceived as non-obtrusive or even human-like. In this way the complexity of AAL devices can be reduced and thus be adopted even by elderly generations with less technical capabilities than future ones.

\section{Threats to Validity}

In this paper we aim at giving an overview of three topics within the AAL domain. We are aware of several threats to validity of this overview. Firstly, the selected literature was chosen based on three keywordsdirectly connected to AAL. Using a broader variety of keywords, a less narrow overview of the AAL domain could have been achieved. In this paper the selected literature was included when it regarded the three geographic regions Europe, Asia and North America. This choice was based on the necessity of these regions of AAL due to the demographic transition. By defining other motives for AAL also another impression of this filed could have been gained.

\section{Conclusion}

In this paper, we intended to give an overview of the Ambient Assisted Living domain. Therefore, we firstly defined the necessity for the development of AAL solutions. Furthermore, we differentiated the term Ambient Assisted Living from other e-health domains and gave insight into the evolution of AAL devices. To provide an overall picture of the field of AAL the three topics: current applications, end-users and acceptance have been defined as relevant and were investigated in this paper. Regarding these three topics we conclude:

i. Various applications exist in the AAL domain to enable independent living in the users' homes. The most common application is the smart home. To provide custom AAL services various sensors are deployed. Also, robots are able to assist the resident in multiple ways.

ii. Due to an ageing society mostly, elderlies are targeted by AAL devices. The devices are also targeted at a specific loss of independence. Next to activities of daily living, AAL assists with social or cognitive impairments. Also, not impaired people might be end-users of AAL iii. The acceptance of AAL among the mostly elderly endusers is mainly positive. The price and the disregarding of user needs during the development are the main hindrance of a broader adoption. A usercentered design is a solution for that.

\section{References}

1. (2019) World Population Prospects 2019, Department of Economic and Social Affairs. United Nations 2019 141: 49-78.

2. Natalie Greene Taylor, B Subramaniam M, Waugh A (2015) Transforming the Future of Ageing. SAPEA: 294.

3. (2017) Asia: At Risk of Growing Old before Becoming Rich?', Regional economic outlook. Asia and Pacific: preparing for choppy seas. International Monetary Fund (April).

4. (2020) World Population Prospects - Population Division - United Nations (2020) population.un.org.

5. Berger H, Dabla Norris E, Sun Y (2019) Macroeconomic of Aging and Policy Implications. Group of 20 IMF Staff Note.

6. (2018) Beschäftigte in der Pflege (Pflegekräfte nach SGB XI). BMG bundesgesundheitsministerium.de.

7. Martinez Fernandez C (2012) Demographic Change and Local Development: Shrinkage, Regeneration and Social Dynamics. OECD Demographic Change and Local D e velo p m e n t : Shrinkage, Regeneration and Social Dynamics: 1- 309.

8. Fischer F (2016) eHealth in Deutschland. eHealth in Deutschland.

9. Abschlussfassung (2016) Studie im Auftrag des Bundesministeriums für Gesundheit.

10. Blackman S, Claudine Matlo, Charisse Bobrovitskiy, Ashley Waldoch, Mei Lan Fang, et al. (2016) Ambient Assisted Living Technologies for Aging Well: A Scoping Review. Journal of Intelligent Systems 25(1): 55-69.

11. Cowan D, Turner Smith A (1999) The Role of Assistive Technology in Alternative Models of Care for Older People. With Respect to Old Age 2: 325-346.

12. Cook DJ, Augusto JC, Jakkula VR (2009) Ambient intelligence: Technologies, applications, and opportunities. Pervasive and Mobile Computing 5(4): 277-298.

13. Rashidi P, Mihailidis A (2013) A survey on ambient-assisted living tools for older adults. IEEE Journal of Biomedical and Health Informatics 17(3): 579-590.

14. Byrne CA, Collier R, Ohare GMP (2018) A review and classification of assisted living systems. Information (Switzerland) 9(7): 1-24.

15. Al Shaqi R, Mourshed M, Rezgui Y (2016) Progress in ambient assisted systems for independent living by the elderly. Springer International Publishing 5(1).

16. Cedillo P, Cristina Sanchez, Karina Campos, Alexandra Bermeo (2018) A Systematic Literature Review on Devices and Systems for Ambient Assisted Living: Solutions and Trends from Different User Perspectives. IEEE: 59-66.

17. Li R, Lu B, Mc Donald Maier KD (2015) Cognitive assisted living ambient system: a survey. Digital Communications and Networks 1(4): 229-252.

18. (2019) CoachMyLife - AAL Program aal-europe.eu.

19. (2012) AIB - AAL Programme aal-europe.eu.

20. Adib F, Hongzi Mao, Zachary Kabelac, Dina Katabi, Robert C Miller, et al. (2015) Smart homes that monitor breathing and heart rate. Conference on Human Factors in Computing Systems - Proceedings: 837-846.

21. Haux R, Sabine Koch, Nigel Lovell, Michael Marschollek (2016) HealthEnabling and Ambient Assistive Technologies: Past, Present, Future. Yearbook of medical informatics: S76-S91. 
22. Alexandru A, Ianculescu, M (2017) Enabling assistive technologies to shape the future of the intensive senior-centred care: A case study approach. Studies in Informatics and Control 26(3): 343-352.

23. Pawar AB, Ghumbre S (2017) A survey on IoT applications, security challenges and counter measures. International Conference on Computing. Analytics and Security Trends, CAST 2016 IEEE: 294-299.

24. TRON Intelligent House (no date) tronweb.super-nova.co.jp.

25. Erden F, Senem Velipasalar, Ali Ziya Alkar, A Enis Cetin (2016) Sensors in Assisted Living: A survey of signal and image processing methods. IEEE Signal Processing Magazine IEEE 33(2): 36-44.

26. ALMA - AAL Programme (no date) aal-europe.eu.

27. Bio Harness | BIOPAC (no date).

28. Yamazaki T (2007) The ubiquitous home. International Journal of Smart Home 1(1): 17-22.

29. ALIAS - AAL Programme (no date) aal-europe.eu.

30. Pollack ME, Laura Brown, Dirk Colbry, Cheryl Orosz, Bart Peintner, et al. (2002) Pearl: A mobile robotic assistant for the elderly. Architecture 2002: 85-91.

31. PARO Therapeutic Robot (no date) parorobots.com

32. Weiss C (2013) Unterstützung Pflegebedürftiger durch technische Assistenzsysteme: 1-144.

\section{ISSN: 2574-1241}

DOI: 10.26717/BJSTR.2020.30.004949

Robert Stodczyk. Biomed J Sci \& Tech Res

CC (i) This work is licensed under Creative

Submission Link: https://biomedres.us/submit-manuscript.php
33. Ni Q Hernando ABG, De la Cruz IP (2015) The elderly's independent living in smart homes: A characterization of activities and sensing infrastructure survey to facilitate services development. Sensors (Switzerland) 15(5): 11312-11362.

34. Ranasinghe S, Al Mac Hot F, Mayr HC (2016) A review on applications of activity recognition systems with regard to performance and evaluation. International Journal of Distributed Sensor Networks 12(8).

35. (2015) European Commission Economy Series. The Ageing Report 2015

36. Mahoney FI, Barthel DW (1965) Functional Evaluation: The Barthel Index. Maryland State Medical Journal (14): 56-61.

37. Wallace M (2008) Katz Index of Independence in Activities of Daily Living ( ADL ) Katz Index of Independence in Activities of Daily Living INDEPENDENCE : DEPENDENCE. American journal of nursing, 108(4): 67-71.

38. AAL S (2019) IEC SyC AAL SMB/6784/R: 9-27.

39. Eberhardt B, Fachinger U, Henke KD (2010) Better health and ambient assisted living (AAL) from a global, regional and local economic perspective. International Journal of Behavioural and Healthcare Research 2(2): 172.

40. Deutsche Telekom AG (2018) Schlussbericht DAAN - Design Adaptiver Ambienter Notifikationsumgebungen.

41. Künemund H, Hrsg UF (2018) Alter und Technik: Sozialwissenschaftliche Befunde und Perspektiven.

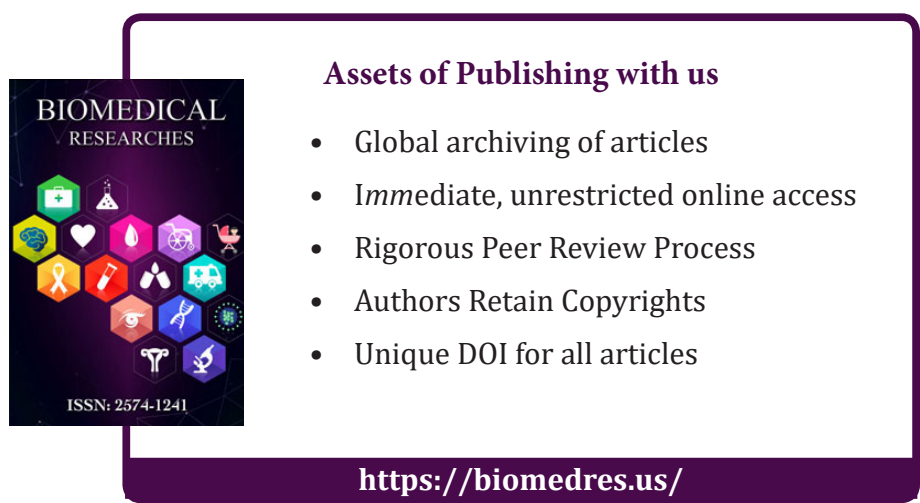

